\title{
AGN feedback in details and in surveys
}

\author{
Alexis Finoguenov ${ }^{1,2}$ \\ ${ }^{1}$ Max-Planck-Institute for Extraterrestrial Physics (MPE), Giessenbach Str., Garching, \\ D-85748, Germany \\ ${ }^{2}$ University of Maryland, Baltimore County, 1000 Hilltop Circle, Baltimore, MD 21250, USA \\ email: alexis@mpe.mpg.de
}

\begin{abstract}
I will review the new details on AGN feedback revealed by recent Chandra observations of M84 and present its theoretical modeling. Using the results of COSMOS survey I will present the direct measurement of halo occupation statistics for radio galaxies.
\end{abstract}

